\title{
A Prediction Model for Population Dynamics of Cotton Pest (Thrips tabaci Linde) using Multilayer-Perceptron Neural Network
}

\author{
Jyothi Patil \\ Scholor ,CMJ University \\ Karnataka,India
}

\author{
V.D.Mytri, PhD. \\ Shetty Institute of technology \\ Karnataka,India
}

\begin{abstract}
India's agricultural sector faces a series of problems when it comes to increasing crop productivity. Despite the efforts of researchers to discover productive agricultural practices, crop yield has not been the most pleasing, and one global reason stated for this poor crop yield is the insect pests. Predicting in advance the occurrence of peak activities of a given pest could enable the development of a suitable pest control mechanism that would initiate better production. Researchers have attempted to comprehend the pest population dynamics by applying analytical and other techniques on pest surveillance data sets. In this paper, An intelligent system for effectual prediction of pest population dynamics of Thrips Tabaci Linde (Thrips) on cotton (Gossypium Arboreum) crop is presented. The raw data used in the proposed system was obtained from the College of Agriculture, Raichur, India. Initially, the raw (pest surveillance) data is prepared by 1) Data preprocessing 2) Normalization and 3) Data transformation. The feed forward Multi-Layer Perceptron (MLP) Neural Network with backpropagation training algorithm is employed in the design of the intelligent system. The neural network is trained and tested with the data prepared. The experimental results portray the effectiveness of the proposed system in predicting pest population dynamics of Thrips on cotton crop. Moreover, a comparative analysis is performed between the proposed system and two of the existing works. The results showed that the proposed system based on feed forward neural networks was best suited for effective pest prediction.
\end{abstract}

\section{General terms}

Population Dynamics of Cotton Pest Thrips

\section{Keywords}

Pest; Pest Population Dynamics; Pest Surveillance Data; Prediction; Cotton Crop; Thrips Tabaci Linde; Intelligent System; Multi-layer Perceptron Neural Network (MLPNN); Backpropagation training Algorithm.

\section{INTRODUCTION}

The developments in information technology (IT) have enhanced all fundamentals of our life, in the last decade. Current advancement in IT has facilitated the course of enormous information (text, image, sound and video) repositories with minimal down-time. Perhaps, the stored information can be mined out concurrently by millions of users [2]. Data mining techniques are used for the extraction of valuable data from large databases and in addition the simulation technology can be utilized to forecast the upcoming [3]. These IT-based advancements has showed the way for innovative opportunities to increase the utilization and performance of livelihood technologies like agriculture, education, library, health and medical services, and artesian technologies [1].
A significant part of Indian population living in the rural areas earns their living directly or indirectly from farming and agriculture, and therefore, agriculture plays a significant role in Indian economy. Indian farming is encountering several issues in the endeavor to increase crop productivity. Despite the number of successful researches on new agricultural technologies related with crop cultivation majority of the farmers are still not able to produce upper-bound yield owing to several other reasons. One main reason is that the expert/scientific guidance related to crop cultivation does not arrive at the farming community at the right moment [4]. Unquestionably, India has invaluable amount of agricultural knowledge and skill. However, there exists a huge information gap between research and practice. Indian farmers need correct expert advice at the right moment in order to be productive and competitive [5]. A main restraint to crop productivity comes in the type of insect pests.

The environment includes insects, mildew, rodents, bacteria and other such organisms. While a few of these are beneficial to people in various ways, others are harmful in nature [6]. A main complexity in resolving the pest management issue is the inadequate knowledge about the characteristics of the pest dynamics. This directs to a situation called as inadequate control [7]. Various researchers have suggested that the increasing insect pest and diseases in agro-ecosystems is an outcome of alterations made to agricultural practices since World War II. For example, the utilization of pesticides has been on a steady rise since that time and proof illustrates that such needless use of agrochemicals in mixture with intensifying monocultures has increased pest problems [8].

Research works are intended to understand pest dynamics with the use of analytical and related procedures on pest surveillance data sets. These forecasts would help the farmers in the pest management programs as they are capable of avoiding unwarranted chemical pesticides being sprayed on the crop, thereby stopping crop losses and also bringing about improved environment quality [9]. Normally, the farmers come across severe losses on their crops due to pests. To protect the farmers from the losses and to assist them in better production, successful pest prediction methodologies are expected. It is essential that a competent pest control methodology would be able to forecast the pest dynamics accurately. Thus, the need for creating an applicable and functionally viable model to forecast the pest incidence is the current requirement [10].

Even though a greater part of risks in the agricultural sector are controllable, a few of them are unavoidable. The temporal and spatial unpredictability of rainfall, temperature and evaporation, and change in climate conditions like atmospheric carbon dioxide levels, comprise the agro meteorological dangers common in farming. Moreover, despite causing a heavy impact directly on plant growth and development, these aspects also exhibit an indirect outcome by influencing the life cycles of plant diseases and pests. Furthermore, these are also responsible for intensely 
disturbing the pest control measures as observed when a sudden rainfall event results in dilution or early hydrolysis of a surface pesticide, or when hail damage paves way for mould, bacterial or insect attack. It is essential that the integrated pest management (IPM) should consider such risks, if crop damage is to be minimized [11].

The insufficient knowledge about the issues influencing pest population dynamics is one of the troubles involved in pest management. It is essential to predict the peak activities of the pest exactly so as to make the pest management effective and efficient. Agricultural scientists collect the pest surveillance data linked to agricultural operations about crops, farming practices and other weather parameters in order to understand the pest dynamics. Several details of pest incidence, climatic conditions, soil details, irrigation, agricultural practices, etc are enclosed in these databases [7]. Intellectual decisions can be made with these databases that are loaded with valuable and hidden information. Classification and prediction are the two forms of data analysis that can be used to extract models explaining the future trends. Yet, farmers require a functionally practical model for pest prediction for efficient and effective pest management.

A top of the list pest that badly affects the Cotton crop resulting in yield loses is Thrips tabaci Linde (Thrips). Numerous amounts of ecological and physiological features influence its severity in diverse scenarios. Normally, the climatic data takes a stable seasonal pattern that repeats almost every year. Hence, these pests are also likely to display a particular model in the population dynamics. Nevertheless, the pest can possibly include volatile biological events. This intrinsic character of Thrips makes it difficult to its predict population dynamics with the support of conventional prediction models.

In this paper, An intelligent system based on Neural Networks (NN) for predicting the pest population dynamics of Thrips tabaci Linde is presented with the intention of improving cotton crop productivity. A comparative analysis between the proposed intelligent system and two of the existing works [7, 9] available in the literature for pest prediction is performed. The proposed system predicts the population dynamics of the Thrips tabaci Linde pest on the cotton crop (Gossypium arboreum) with the aid of the pest surveillance data attained from College of Agriculture, University of Agricultural Sciences, Raichur, India. The primary step involved in the proposed research is data preparation. The pest surveillance data is prepared by the following set of processes namely, data reduction, elimination of null valued tuples and normalization. The resultant data is then utilized in the training of the neural network, standard feed forward Multilayer perceptron (MLP) neural network structure with Backpropagation training algorithm. The prediction accuracy of the proposed system for Advance (0), Advance (1) and Advance (2) are demonstrated by the experimental results. Lastly, the performance (correlation and \% of hits and misses) of the proposed system was evaluated by a comparative analysis. The comparison results showed that the proposed system achieved better prediction accuracy than its relative counterparts chosen for the study.

The rest of the paper is organized as follows. A brief review of the works related to the proposed intelligent system is presented in Section 2. A concise description of the Cotton crop and its pests is provided in Section 3. Section 4 describes the pest surveillance data set chosen for the study. The proposed intelligent system for predicting the population dynamics of Thrips pest in Cotton crop is explained in Section 5. Section 6 describes the experimental results and comparative analysis of the proposed research. Conclusions are summed up in Section 7.

\section{REVIEW OF EXISTING WORKS}

Literature presents with a number of researches for the prediction of pest population dynamics in varied types of crops. The motivation drive is from some of those previous works available in the literature. A selected few noteworthy contributions are given below:

Ch. Pavan Kumar et al. [10] made use of neural networks theory and Bayesian classification on pest surveillance data set of Yellow stem borer on rice crop to study the pest dynamics. The data set comprises of details regarding weather, pest incidence and other farm related details and cover over 12 years. The pest prediction methodology which makes use of neural networks using Levenberg-Marquardt algorithm to guide the network has been explained and the experimental results were considered. The successful prediction of pest attack incidences in advance by a week by the network method was illustrated by the results. Additionally, they used Bayesian classification to compare its results with the predicted results of neural network.

The worldwide circulation of two pest species- Ceratitis capitata (Wiedemann), the Mediterranean fruit fly, and Lymantria dispar (L.), the gypsy moth was expected based on climatic factors by Muriel Gevrey et al. [12]. Classical statistical models or eco-climatic assessment models like CLIMEX have been mainly frequently used in the investigation of the distribution patterns of insect pests. They modeled the distribution of each species by using an artificial neural network, the multilayer perceptron, trained using the backpropagation algorithm. To model the distribution of each species, the data matrix utilized was separated into three data sets that proposed to 1) develop and train the model, 2) validate the model and prevent over-fitting, and 3) test each model on novel data. The global distribution of each species had a high percentage of correct predictions for the three data sets; for Mediterranean fruit fly giving 95.8, 81.5, and 80.6\% correct predictions respectively and for the gypsy moth giving $96.8,84.3$, and 81.5 , respectively.

Gang Liu et al. [13] utilized the MATLAB neural network toolbox to build up the prediction system about fruit tree diseases and insect pests based on Backpropagation neural network in accordance with the weather information and occurrence status of fruit tree diseases and insect pests in orchards. The history record data was utilized to train the data. Finally, they planned to compare the predicted value with the actual value by fixing the ring spot, a fruit tree disease, as the research object. It can be certain from the results that the system executed accurately, swiftly and robustly. The applications that predict fruit tree diseases and insect pests can utilize the system.

An effort has been made to compare the efficiency of the prediction systems using the Regression modeling, Bayesian analysis and neural network techniques by Dangeti Ravi Shankar et al. [7]. It was achieved by understanding the pest dynamics of Spodptera Litura on the groundnut crop with the data provided by ICRISAT. It was found that Neural Network systems offered results with a very high degree of accuracy which suits the building of a pest prediction system for groundnut crop. Rajat Gupta et al. [9] have made an effort to understand pest population dynamics using Neural Networks by analyzing pest surveillance data set of Helicoverpa armigera or Pod borer on chickpea (Cicer arietinum L.) crop. The results showed that neural network method successfully predicts the pest attack incidences for one week in advance. 


\section{COTTON CROP AND ITS PESTS}

Primarily, cotton is an agricultural crop, but was also found to be grown wild. Among the 30 available species, only 4 are utilized in supplying the world market with cotton. The conventional and systematic assessment of cotton for insects and the associated damage is the cotton insect scouting. This section explains about the cotton crop and the pests related to it [14].

\subsection{Cotton Crop}

Cotton is a soft, staple fibre that grows into a boll around the seeds of the cotton plant, a shrub that is extensive at tropical and subtropical regions around the world including, America, India and Africa. Gossypium Arboretum is the scientific name of the cotton tree. Harappan civilization had cultivated cotton for producing cotton textiles way back in 2000 BC [14]. Normally, it is utilized in the making of soft textile; the most widely utilized natural-fibre cloth and is created after the fibre is spun into yarn or thread. Its English name, circa 1400, was derived from the Arabic (al) qutn قُطنْ , which translates to cotton.

\subsection{The Pests}

The main reason for the loss of cotton yield is the insect pests. It is predicted that the loss occurred due to the insect infections would be up to $15 \%$ of world annual production. More than 1300 diverse species of insect pests affecting the crops have been found. A few most affecting pests would include Pink bollworm (Pectinophora gossypiella), boll weevil (Anthonomus grandis), Egyptian (spiny) bollworm (Earias insulana), red bollworm (Diparopsis castanea), Cotton stainers (Dysdercus superstitious), white flies (Bemisia gossypiella), Thrips tabaci Linde (Thrips) and Aphis gossypii Glover (Aphids). The most extensive of the aforesaid species' found in cotton fields are explained below.

Thrips: This cotton pest is normally found to be in yellow, brown, or black in color. Thrips generally measure less that 2 $\mathrm{mm}$ in length. The adults contain two pairs of narrow wings fringed with long hairs while a few species are devoid of wings during winter. Thrips live on the weeds during the off season. Once the seedlings emerge above the ground, they move onto cotton. Male Thrips are infrequent and the parthenogenetic reproduction is followed. Initially, Thrips inhabit on leaves of cotton. Then later, colonies inhabit on bolls. Seedlings infected with Thrips show some symptoms such as slow growth and the leaves are wrinkled, curled upwards and distorted with white shiny patches, rusty appearances in parches developed on undersurface of leaves [15].

\section{DESCRIPTION OF PEST SURVEILLANCE DATA}

The scientists at the College of Agriculture, University of Agricultural Sciences, Raichur, India, collected the observation data to understand the population dynamics of Thrips tabaci Linde (Thrips) pest on cotton crop. The observation data holds a set of weekly recordings regarding the weather and pest incidence at diverse spots in the campus. The recordings range from the year 2003 to 2008. The data set contains 247 week of recordings over a span of 5 years. A brief explanation of the diverse attributes in the surveillance data is as follows:

\section{Standard Week:}

The weeks in a year are mapped into integer values by considering the first week of January as first standard week.

\section{Weather Conditions:}

Minimum Temperature (Min.T $\left({ }^{\circ} \mathbf{c}\right)$ ): The lowest temperature recorded in the College of Agriculture, Raichur campus on the particular week of survey.

Maximum Temperature (Max.T $\left.\left({ }^{\circ} \mathbf{c}\right)\right)$ : The highest temperature recorded in the College of Agriculture, Raichur campus on the particular week of survey.

Humidity: The relative humidity recorded in morning (RH I (\%)) and evening (RH II (\%)) at the College of Agriculture, Raichur campus on the day of survey.

Rainfall (RF ( $\mathbf{m m})$ ): The amount of rainfall recorded on College of Agriculture, Raichur campus in the particular week of survey.

\section{Pest Incidence:}

Jassids/leaf: It is the average number of jassids (leafhoppers) per leaf and the methodology is involved in a given area of $20 \mathrm{sqm}$. Generally, five plants are selected randomly in above mentioned size plot and in each plant three leaves (Top, Middle and Bottom) will be observed for leafhopper number.

In the above said manner the recordings for Thrips, Whiteflies and Aphids are observed.

Ha eggs: Helicoverpa armigera is a bollworm. In each plant, number of eggs present on top three leaves were calculated on five randomly chosen plants and expressed as number of eggs per plant. Similar is the case for Helicoverpa armigera larvae (Ha larvae).

\% rosette flowers: It is nothing but the damaged flowers due to pink bollworm and here number of good flowers and damaged flowers will be calculated and expressed as \% rosette flowers.

\% TFB: Thenumber of fruiting bodies damaged and healthy was recorded on five randomly chosen plants.

\section{INTELLIGENT SYSTEM FOR PEST POPULATION DYNAMICS PREDICTION}

Artificial Intelligence (AI) is defined as the study of ideas that facilitate computers to achieve things that make people appear intelligent [16]. Neural Networks (NN) is one of the most powerful AI techniques that are capable of learning from a set of data [17]. Neural network modeling has been extensively recognized as a dominant means for solving complex, nonlinear biological problems like pest incidence. A standard feed forward Multi-layer perceptron (MLP) neural network structure with Backpropagation training algorithm was employed in the proposed intelligent system for predicting pest population dynamics of Thrips pest in cotton crop. The proposed system makes use of the data obtained from the College of Agriculture, University of Agricultural Sciences, Raichur, India for comprehending the population dynamics of Thrips on the cotton crop. The steps involved in the prediction of the population dynamics of pests are given as follows.

\subsection{Raw Data}

The raw data acquired from the College of Agriculture, University of Agricultural Sciences, Raichur has its values unevenly distributed and noisy. Clearly, one primary criterion to decide on the quality of the results is the quality of the data employed. As the raw data collected for our study is likely to contain noises, it is to be preprocessed so as to, fill in those missing values, smooth the noisy data, identify or remove outliers and resolve inconsistencies. Subsequently, the preprocessed raw data is normalized within a small specified range; Normalization ensures that the system is not influenced inexplicably by high/low attribute values. Lastly, data are 
transformed such that the data can be fed to the feed forward Multi-layer perceptron (MLP) neural network.

5.1.1 Data Preprocessing: Data preprocessing encompasses 1) data reduction and 2) elimination of null values. The input to the data preprocessing phase is the pest surveillance data set chosen for the study.

5.1.1.1 Data Reduction: Probably, the raw data might have empty records for certain dates where data weren't collected because of bad weather or absence of scout. When datasets with empty records are fed to the AI technique employed, it is likely that there is a probability of the data set leading to biased outcomes. One probable solution is to reduce the entire dataset to some form order data with regularized intervals. Here, a single consolidated value is computed for all the individual attributes for a week by averaging the values corresponding to individual days. Thus, reduced data corresponding to every week will not have empty records.

5.1.1.2 Elimination of Null Valued Tuples: Another one important factor that influences decision making is the presence of null values in the dataset. The null values may be either due to the absence of scout, unfavorable weather condition or failure of equipment. All null valued tuples from the reduced dataset are removed.

5.1.2 Z-Score Normalization: The preprocessed data is subjected to Z-score normalization so as to bring about uniform distribution of values among different attribute sets. $\mathrm{Z}$-score normalization is one of the most powerful normalization techniques with the maximum and minimum values of the attributes are unknown. It works based on the mean and standard deviation of the distribution of the attribute data. A brief description of the normalization process is given below [7]:

A value $x$ of attribute $A$ is normalized to $x^{\prime}$ by computing

$$
x^{\prime}=\left(x-A^{\prime}\right) / S A
$$

Where,

$A^{\prime}=$ Mean of the distribution $A$.

$S A=$ Standard deviation of the distribution $A$.

$$
Z-\operatorname{score}(x)=(x-\operatorname{Mean}(x)) / \operatorname{Stddev}(x)
$$

Where,

$\operatorname{Mean}(x)=$ Average of all the values of that particular parameter of distribution ' $x$ '.

$\operatorname{Stddev}(x)=$ Standard deviation applied on ' $x$ '.

5.1.3 Data Transformation: With data preprocessing and normalization, unevenly distributed and noisy data values in the dataset are being removed. Yet, the data before being fed to an AI technique has to be transformed such that the data is suited for predicting the pest population dynamics for the crop. In the proposed system, the preprocessed data set comprises of data corresponding to 4 to 5 years with 52 weeks of data for every year. It would be tedious to use this enormous amount of data to train or test the MLP Neural network. Here, the preprocessed dataset is transformed so that the number of records to be considered reduces significantly, i.e. three months data (i.e. twelve weeks of data) are transformed (grouped) under a single label.

\subsection{Neural Network Architecture}

Neural network can be considered as a set of input/output units where in connections between the units possess a certain weight associated with it [18]. The neural network comprises of the training and testing phase. During the training phase, the neural network learns by regulating the weights so as to be capable of predicting the call label of input samples during the testing phase. Besides their capability to recognize patterns based on the trained data, the neural networks exhibit great resistance towards noisy data. The neural network employed in the proposed intelligent system is a feed forward MLP network.

In feed-forward neural networks, the input to the second layer neurons is the output of the first layer neurons that are fed in a unidirectional fashion (i.e. propagation of the neurons along the reverse direction is not permitted). Multi-layer Perceptron Neural Network (MLPNN) or Multi-layer feed-forward Neural Network (MFNN) is a kind of feed-forward neural network.

The neurons in the input layer perform the only task of distributing the input signal $x_{i}$ to neurons in the hidden layer.

Each neuron $j$ in the hidden layer adds up its input signals $x_{i}$ once it weighs them with the strengths of the corresponding connections $w_{j i}$ from the input layer and determines its output $y_{j}$ as a function $f$ of the sum, given by,

$$
y_{j}=f\left(\sum W_{j i} X_{i}\right)
$$

Where $f$ is simple threshold function (sigmoid or hyperbolic tangent function). The results of the neurons in the output layer are also determined in a similar way. Then, so as to permit a network to accomplish a desired general behavior, the strengths of the connections are regulated by means of a learning algorithm. Generally, two sets of data is employed, one for "training" and the other for "testing". Roughly, about $80 \%$ of the data set is utilized for training and the remaining $20 \%$ for testing.

Training the Neural Network: The neural network is actually trained using the Backpropagation Algorithm. Backpropagation Algorithm is recognized as the most versatile algorithm and it works on the basis of a multilayered feed-forward net [19]. On reception of the input on the first layer neurons, the backpropagation algorithm applies the Linear Combiner and Activation Function to the inputs and generates the output. The output obtained is then propagated to the neurons in the next layer. The next layer will feed forward the data to the layer after it and so on, until the last layer is reached. After the input pattern has been processed by all the layers of the network, the network are adjusted based on the errors recorded (the difference between what is wanted and what is got). The above process is called "backpropagation" and is carried out by adjusting the weights. The backpropagation algorithm trains the network for predicting the correct outputs those acquired from experiments and generated ones. The operation of the typical backpropagation network is given as [20].

1.Transmission of information through the network to the output layer (forward propagation) once the data is presented to the input layer. The time input and output states for each neuron will be set during the course [21].

$$
\left.X_{j}^{[S]}=f\left(I_{j}^{[S]}\right)=f\left(\sum_{i} W_{i j}^{[S]^{*}} X_{i}^{[S-1]}\right)\right)
$$

Where, $X_{j}^{[S]}$ - the current input state of the $\mathrm{j}^{\text {th }}$ neuron in the current $[S]$ layer [23]. 
$I_{j}^{[S]}$ - the weighted sum of inputs to the $\mathrm{j}^{\text {th }}$ neuron in the current layer $[S]$.

$f$ - the sigmoid function.

$W_{i j}^{[S]}$ - the connection weight between the $\mathrm{i}^{\text {th }}$ neuron in the current layer $[S]$ and $\mathrm{j}^{\text {th }}$ neuron in the previous layer $[S-1]$.

2. Determination of the generated global error, which is the summed difference of required and calculated output values of each neuron in the output layer [23].

The Normalized System error $E($ glob $)$ is given by the equation $E(g l o b)=0.5 *\left(r_{k}-o_{k}\right)^{2}, \quad$ Where $\left(r_{k}-o_{k}\right)$ - the difference of desired and computed output values.

3. Computation of the local error values and delta weights for every neuron by back propagating the global error through the network. Delta weights are modified regarding the delta rule that strongly controls the incessant decline of synaptic strength of those neurons that are principally responsible for the global errors [23]. Thus, the regular reduction of global error can be ensured [22].

$$
E_{j}^{[S]}=X_{j}^{[S]} *\left(1.0-x_{j}^{[S]}\right) * \sum\left(e_{k}^{[S+1]} * W_{k j}^{[S+1]}\right.
$$

Where $E_{j}^{[S]}$ - the scaled local error of the jth neuron in the current layer $[S]$ layer.

$$
\Delta w_{j i}^{[S]}=\text { lcoef } * e_{j}^{[S]} * X_{i}^{[S-1]}
$$

Where $\Delta w_{j i}^{[S]}$ - the data weight of the connection between the current neuron and the adjacent neuron [23]. Here, lcoef represents the learning coefficient/ learning constant of the training parameters.

4. Addition of the delta weights to the current weights for updating the Synaptic weights.

The transformed data, each comprising of three months, is fed as training input to the feed forward MLP neural network. The neural network learns from the training dataset by means of the backpropagation training algorithm. Subsequently, when a dataset corresponding pest population dynamics is provided to the trained neural network, it predicts (forecasts) the pest population dynamics of Thrips tabaci Linde (Thrips) pest on cotton (Gossypium Arboreum) crop for the future days (say days, weeks) based on the learnt population dynamics

\section{TEST RESULTS AND ANALYSIS}

This section describes the experimental results of the proposed intelligent system for pest prediction and the comparative analysis of the proposed system with the existing works $[7,9]$.

\subsection{Results on experimentation}

The results obtained on experimentation of the proposed intelligent system are presented in this sub-section. The pest surveillance data set of the cotton crop employed in the proposed system was collected over a period of 5 years (20032008) and comprises of recordings corresponding to 247 weeks. Among the entire surveillance data set, data collected over the three years (2005-2008) are used for training and the rest (2003-2005) are used for testing. Every tuple in the surveillance data set comprises of: Max.T $\left({ }^{\circ} \mathrm{c}\right)$, Min.T $\left({ }^{\circ} \mathrm{c}\right), \mathrm{RF}$ (mm), Rainy Day, RH I (\%), RH II (\%), Thrips/leaf, each representing the weekly mean. Experimenting directly with the raw data set may give very undesirable results and hence the data set is prepared suitably for neural network training. The steps involved in the data preparation include data preprocessing, followed by data normalization and transformation. The entire data set is classified into the training and test data. On training, the neural network learns from the training data inputted to the system. Subsequently, when a test data is provided to the intelligent system, the neural network predicts the pest population dynamics of thrips on cotton crop. One crucial factor in pest prediction is the ability of the system to forecast the likeliness of the occurrence of the pest as early as possible i.e. months or weeks before. One common terminology employed to evaluate the aforesaid factor is advance (i), which represents prediction for the $i^{\text {th }}$ week in advance. The terminologies employed in the experimentation of the proposed system are,

Advance (0) - Given the weather parameter of a particular week, the proposed intelligent system forecasts the pest population dynamics of the same week.

Advance (1) - Given the weather parameter of a particular week, the proposed intelligent system forecasts the pest population dynamics of the next week.

Advance (2) - Given the weather parameter of a particular week, the proposed intelligent system forecasts the pest population dynamics of the second week.

The prediction graphs for different advance (i) values are provided below. Each prediction graph portrays the actual and predicted curves corresponding to the test data.

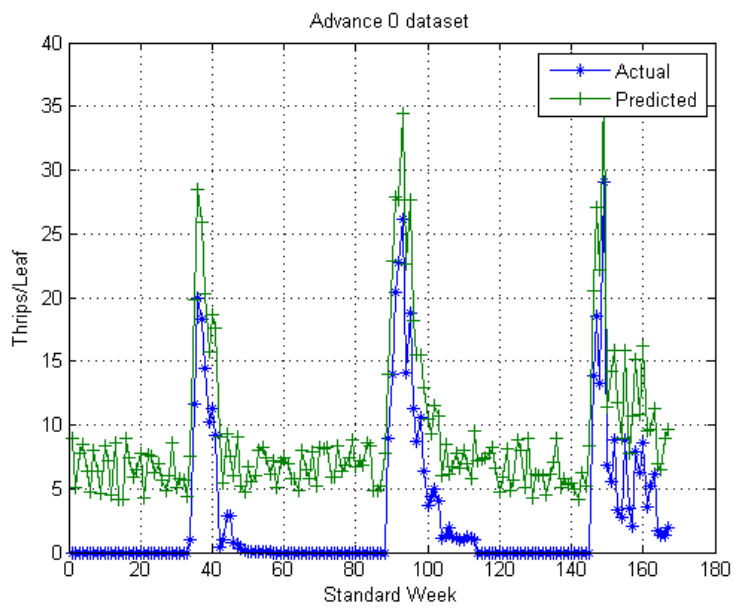

Figure 1. Prediction graph for advance (0) 


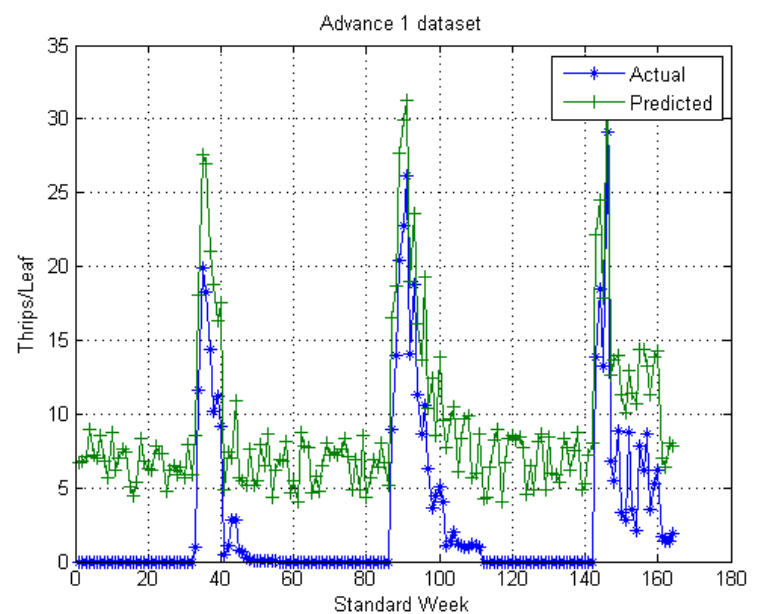

Figure 2. Prediction graph for advance (1)

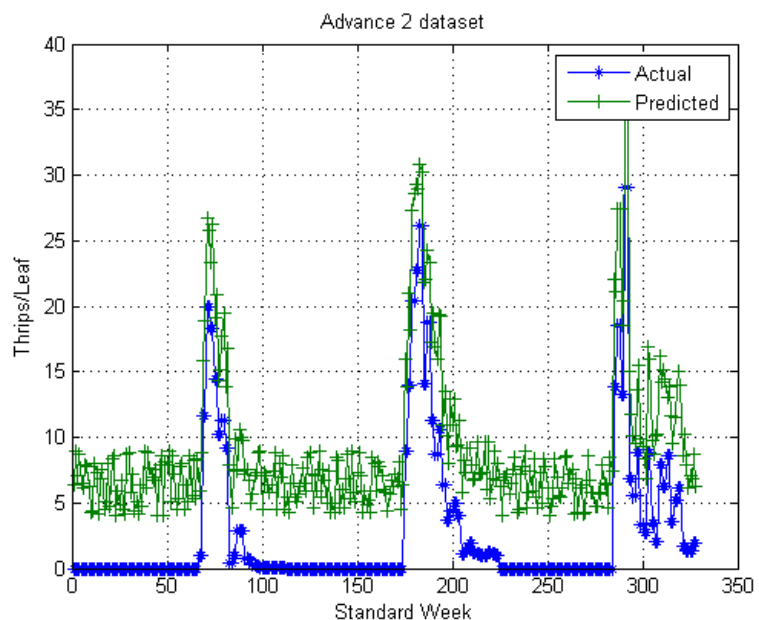

Figure 3. Prediction graph for advance (2)

\subsection{Comparative analysis}

The comparative analysis of two of the existing works [7, 9] with the proposed intelligent system has been illustrated in this section. The parameters that can best illustrate the efficacy of an approach are: Correlation: Measure of the strength and direction of a relationship between the predicted and actual values, Hits: Number of attacks predicted correctly by the system, Misses: Number of attacks left unpredicted by the system. Here, the performance of the proposed intelligent system based on the aforesaid set of three parameters is compared. Table 1 portrays the results obtained 1) Correlation between actual and predicted values 2) \% of Hits and 3)\% of Misses for each of the work included in the study for advance 0 , advance 1 and advance 3 . Hit percentage is defined as the ratio between the number of hits to the total number of test instances provided. Similarly, Miss Percentage is defined as the ratio between the number of misses to the total number of test instances provided.

Table 1. Comparison Results

\begin{tabular}{|c|c|c|c|c|c|c|c|c|c|}
\hline \multirow{2}{*}{ Dataset } & \multicolumn{3}{|c|}{ Correlation } & \multicolumn{3}{c|}{ Hits (\%) } & \multicolumn{3}{c|}{ Misses (\%) } \\
\cline { 2 - 11 } & $\begin{array}{c}\text { Proposed } \\
\text { system }\end{array}$ & $\begin{array}{c}\text { Gupta et } \\
\text { al. [9] }\end{array}$ & $\begin{array}{c}\text { Shankar } \\
\text { et al. [7] }\end{array}$ & $\begin{array}{c}\text { Proposed } \\
\text { system }\end{array}$ & $\begin{array}{c}\text { Gupta et al. } \\
{[9]}\end{array}$ & $\begin{array}{c}\text { Shankar et } \\
\text { al. [7] }\end{array}$ & $\begin{array}{c}\text { Proposed } \\
\text { system }\end{array}$ & $\begin{array}{c}\text { Gupta et } \\
\text { al. [9] }\end{array}$ & $\begin{array}{c}\text { Shankar } \\
\text { et al. [7] }\end{array}$ \\
\hline advance(0) & 0.96633 & 0.91 & 0.87 & 88.6 & 87.1 & 85 & 11.4 & 12.9 & 15 \\
\hline advance(1) & 0.97078 & 0.96 & 0.9 & 90.9 & 90 & 90 & 9.1 & 10 & 10 \\
\hline advance(2) & 0.96957 & 0.91 & 0.89 & 93.9 & 93.1 & 90.5 & 6.1 & 6.9 & 9.5 \\
\hline
\end{tabular}


The comparison results shown in Table 1 are plotted as graphical representation in the following figures. Figure 4, 5 and 6 depict the comparison charts based on correlation coefficients, Hits (\%) and Misses (\%) respectively.

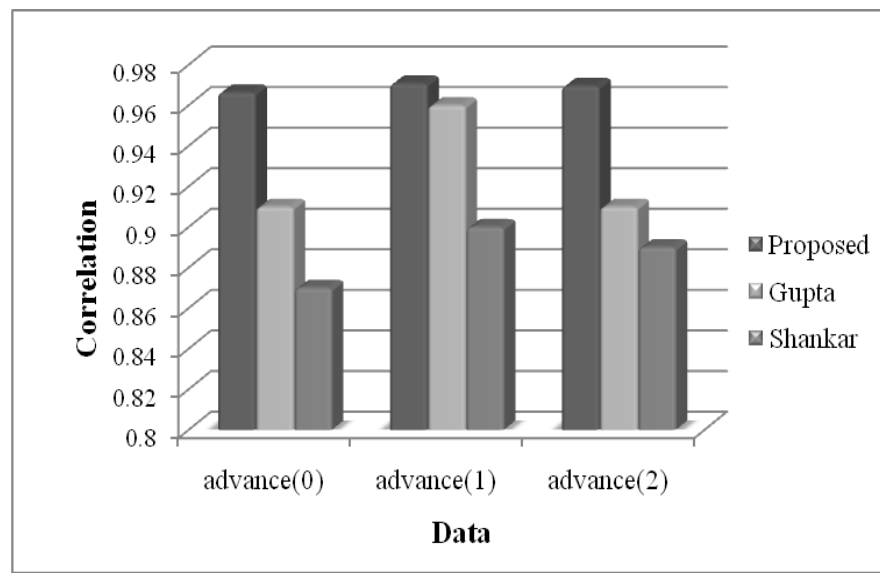

Figure 4. Correlation graph for the three sytems compared

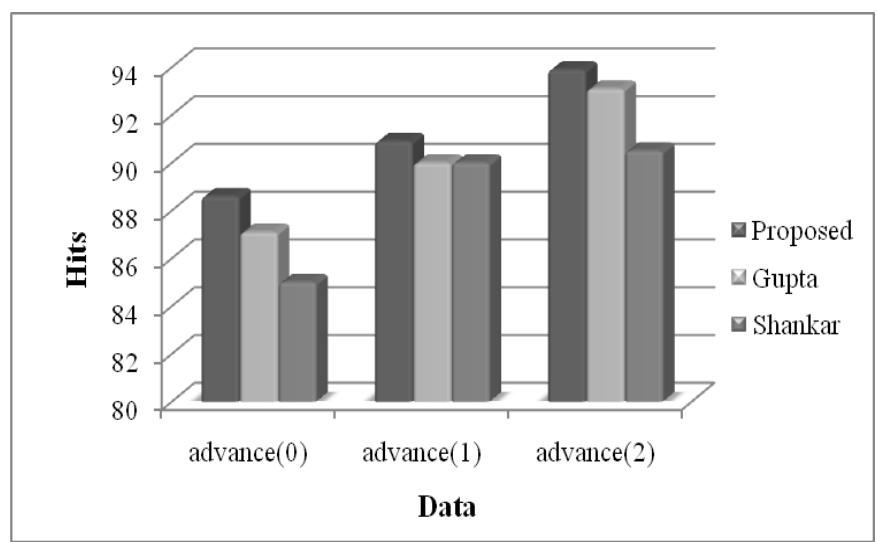

Figure 5. Hits graph for the three sytems compared

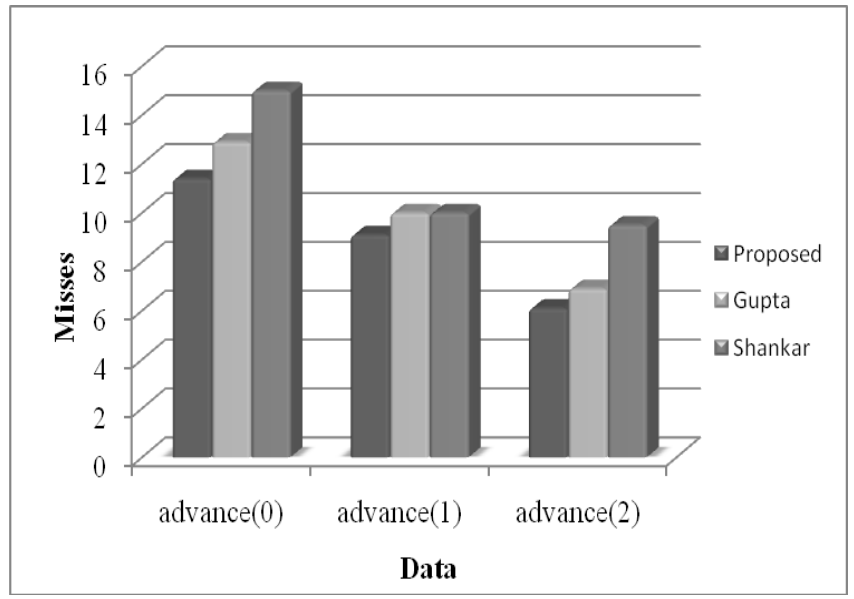

Figure 6. Misses graph for the three sytems compared

All the three pest prediction systems considered for study are meant to predict different pest types for different crops. Also, the three systems make use of three different Neural Networks for its design. The results depicted above just gives an abstract idea of the performance (prediction accuracy) of each of the three systems considered. The results and the graphs depicted clearly shows that the proposed intelligent system for prediction of pest population dynamics of Thrips tabaci Linde (Thrips) on cotton (Gossypium Arboreum) crop performs relatively efficient when compared to the other two systems considered.

\section{CONCLUSION}

In this paper, we have presented an intelligent system for effectual prediction of pest population dynamics of Thrips tabaci Linde (Thrips) on cotton (Gossypium Arboreum) crop. The raw (pest surveillance) data used in the proposed system was obtained from the College of Agriculture, Raichur, India. The pest surveillance data is prepared by the following set of processes namely, data reduction, elimination of null valued tuples and normalization. The resultant data is then utilized in the training of the neural network, standard feed forward Multi-Layer Perceptron (MLP) neural network structure with Backpropagation training algorithm. The performance of the proposed system was evaluated by a comparative analysis. The comparison results showed that the proposed system achieved better prediction accuracy than its relative counterparts chosen for the study. The predictions of the proposed system can be made use of for improving the crop productivity and thereby entrusting new life for Indian farmers.

\section{REFERENCES}

[1] P. Krishna Reddy, "A framework of information technology-based agriculture information dissemination system to improve crop productivity", Current Science, vol. 88 , no. 12,25 June 2005 .

[2] Raj Reddy, "Computing - The next 10 years", talk presented at Georgia Tech 10th anniversary convocation, April 6, 2001 (The presentation is available at http://www.rr.cs.edu/rrlong.html).

[3] Gio Wiederhold, "Information systems that also predict into future", Lecture Notes in Computer Science 2544, Springer_Verlag, pp 1-14, 2002.

[4] B. V. Ratnam, P. Krishna Reddy and G. S. Reddy, "eSagu: An IT based personalized agricultural extension system prototype - analysis of 51 Farmers' case studies", International Journal of Education and Development using ICT, vol. 2, No. 1, 2006.

[5] Vayyavuru Sreenivasulu and H.B. Nandwana, "Networking of Agricultural Information Systems and Services in India", INSPEL vol. 35, No. 4, pp. 226-235, 2001.

[6] Hong Zhang, Paul Georgescu, Lansun Chen, "An impulsive predator-prey model of integrated pest management", CEU, Dept. of Mathematics, 2007.

[7] Dangeti Ravi Shankar, Akhil Kodali, Pradeep Beerla, D.V.S Mohan Nimai, "Neural Networks in Cultivation", Fourth International Conference on Information Technology, pp. 255-260, 2007.

[8] Miguel A. Altieri, Clara I. Nicholls, "Soil fertility management and insect pests: harmonizing soil and plant health in agroecosystems", Soil \& Tillage Research, pp. 203-211, 2003.

[9] Rajat Gupta, BVL Narayana, P.Krishna Reddy, G.V.Ranga Rao, "Understanding Helicoverpa armigera Pest Population Dynamics related to Chickpea Crop 
Using Neural Networks", Third IEEE International Conference on Data Mining, pp. 723- 726, 2003.

[10] Ch. Pavan Kumar V. Pavan Kumar, P.Krishna Reddy, G. Rama Murthy, "Yellow Stem Borer (pest) Attack Prediction System for Rice crop using Neural Networks and Bayesian Classification", International Institute of Information Technology, 2004.

[11] A.K.S. Huda, T. Hind-Lanoiselet, C. Derry, G. Murray and R.N. Spooner-Hart, "Examples of coping strategies with agro meteorological risks and uncertainties for Integrated Pest Management", ARCP, 2007.

[12] Muriel Gevrey and S. P. Worner, "Prediction of Global Distribution of Insect Pest Species in Relation to Climate by Using an Ecological Informatics Method", Journal of Economic Entomology vol:99, no:3, pp:979-986. 2006.

[13] Gang Liu , Hongyan Shen, Xuehong Yang and Yinbing Ge, "Research on Prediction about Fruit Tree Diseases and Insect Pests Based on Neural Network ", IFIP International Federation for Information Processing, Springer Boston, pp: 1861-2288, Volume 187,2005.

[14] Abedin, Sultanul, "Gossypium arboreum", in Ali, S.I.; Qaiser, M., Flora of Pakistan, 130, St. Louis: University of Karachi \& Missouri Botanical Garden, pp. 30, 1979.

[15] Dr. S. Vennila, Dr. V.K. Biradar, Mr. M. Sabesh, Dr. O. M. Bambawale, "Know your Cotton Insect Pest THRIPS", Central Institute of Cotton Research, Nagpur, Crop protection folder series, 3 of 11, March 2007.

[16] P. Cunningham, J. Carney, and S. Jacob, "Stability problems with artificial neural networks and the ensemble solution", Artificial Intelligence in Medicine, vol. 20, no.3, pp.217-225, 2000.

[17] Y. Hayashi, R. Setiono, and K. Yoshida, “A comparison between two neural network rule extraction techniques for the diagnosis of hepatobiliary disorders", Artificial Intelligence in Medicine, vol. 20, no. 3, pp. 205-216, 2000.

[18] Martin T. Hagan, Howard B. Demuth, Mark H. Beale, "Neural Network Design", chapters 1-12, Thomson learning, 2001, ISBN: 0- 9717321-0-8.SavkovicStevanovic, "Neural networks for process analysis and optimization: modeling and applications", Computers \& chemical engineering, Vol. 18, No. 11-12 (14 ref.), pp. 1149-1155, 1994

[19] Savkovic-Stevanovic, "Neural networks for process analysis and optimization: modeling and applications", Computers \& chemical engineering, Vol. 18, No. 11-12 (14 ref.), pp. 1149-1155, 1994.

[20] MR Narasingarao, R Manda, GR Sridhar, K Madhu, AA Rao, "A Clinical Decision Support System Using Multilayer Perceptron Neural Network to Assess Well Being in Diabetes", 36th Annual Scientific Meeting of the Research Society for the Study of Diabetes in India, 21, 22 and 23 November 2008.

[21] Venkatesan P, Anitha S., "Application of a radial basis function neural network for diagnosis of diabetes mellitus", Current Science 2006; 91:1195-9.

[22] Raghu Korrapati, "A Bayesian Model Framework to determine patient compliance in Glaucoma cases", Publisher: Iuniverse Inc. ISBN: 9780595368396. 